\title{
Acta
Biochimica
Polonica
}

Vol. 44 No. $2 / 1997$

$251-258$

QUARTERLY

\section{Expression of the yeast NAM9 gene coding for mitochondrial ribosomal protein*}

\author{
Magdalena Boguta ${ }^{\circledR}$, Agnieszka Chacińska, Marcin Murawski \\ and Barbara Szcześniak
}

Institute of Biochemistry and Biophysics, Polish Academy of Sciences, A. Pawińskiego 5A, 02-106 Warsaw, Poland

Received: 17 January, 1997

Key words: yeast, Saccharomyces cerevisiae, mitochondria, ribosome

\begin{abstract}
We studied expression of the NAM9 gene of Saccharomyces derevisiae that was previously reported to code for a mitochondrial ribosomal protein. Increase in NAM9 gene dosage is accompanied by the increase in both nitRA and protein. The levels of the NAM9 transcript and protein are both reduced in cells growing on glucose as compared to cells growing on galactose as a carbon source. Nam9p accumulates to the same level in $r h o^{\circ}$ and $r h o^{+}$cells. These results confirm previous data indicating diverse regulation of different mitochondrial ribosomal protein genes and suggest that expression of $\mathrm{Nam9p}$ is not co-ordinated with the expression of other mitochondrial ribosomal components.
\end{abstract}

Expression of numerous ribosomal genes must be perfectly co-ordinated to achieve balanced accumulation of ribosomal components enabling proper assembly of the subunits. In Escherichia coli this co-ordination is achieved at the level of translation through an autogenous feedback mechanism where the accumulated ribosomal protein is the repressor of its own synthesis [1]. In contrast, in the yeast Saccharomyces cerevisiae expression of genes coding for proteins of cytoplasmic ribosomes is co-ordinated at the level of transcription by global regulators Abflp and Raplp [2]. The rate of synthesis of individual proteins could be additionally modulated at the level of splicing, mRNA stability, translation and degradation of proteins synthesised in excess [3].

In mitoribosomes of $S$. cerevisiae the two ribosomal RNAs and a single protein of the
37S subunit are encoded by mitochondrial DNA. The remaining 70-80 mitoribosomal proteins are nucleus-encoded, synthesised in the cytoplasm and transported to mitochondria [4]. Thus genęs in both mitochondria and the nucleus must be expressed in a concerted manner to ensure the production of equimolar amounts of mitoribosomal components. Moreover, the synthesis rates have to be coordinately regulated in response to carbon source. Until now 28 nuclear genes of $S$. cerevisiae coding for mitochondrial ribosomal proteins have bejen described. Data on the regulation of expression were reported for 12 mitoribosomal gënes (for references see [5]). The results of thèse studies do not yet allow to construct a consistent model of the regulation of mitoribosomal genes expression.

We studied the expression of the NAM9 gene of S. cerevisiae. Nam9p was previously

*This work was supported by the State Committee for Scientific Research grant No. 6P04A06208.

To whom correspondence should be addressed. 
reported as a mitoribosomal protein based on phenotypes of mutant as well null alleles of NAM9, immunological properties of $\beta$-galactosidase-Nam9 fusion protein and, finally, sequence homology to the ribosomal protein S4 of bacteria, chloroplasts and eukaryotes $[6,7]$. This paper presents experiments where extra copies of the NAM9 gene were introduced into yeast cells to determine the extent to which the expression of Nam9p is co-ordinated with the expression of other mitoribosomal proteins. Transcription and translation of NAM9 was studied in response to carbon source, the presence of mitochondrial genome and mutation within the NAM9 locus.

\section{MATERIALS AND METHODS}

Strains, media and growth conditions.

The $S$. cerevisiae strains used in this study were: KL14-4A MAT a his1 trp2 (P.P. Słonimski laboratory), MB43-15C MAT $a$ NAM9-1 ura3-1 leu2-3 his3 [7] and W303-1B MAT $\alpha$ ura3-1 leu2-3 his 3 trp1 ade 2 can $^{R}$ (Ro Rotstein laboratory). Derivatives of $r h o^{\circ}$ were constructed as described in literature [8]. Rich yeast media YPG and YPGALA, containing respectively $2 \%$ glucose or $2 \%$ galactose, as well as WO-ura and WO-leu minimal medium, supplemented with $2 \%$ glucose or $2 \%$ galactose and the appropriate amino acids, were described previously [6].

Plasmids. pH13 and pAD6 are centromeric plasmids, carrying respectively NAM9 and NAM9-1 alleles [7]. pFL-NAM9 is a multicopy plasmid encoding NAM9; pFL-NAM9-1 and YEp-NAM9-1 are multicopy plasmids encoding the mutant NAM9-1 allele [9]. Multicopy plasmid are derivatives of Yep351 [10] and pFL44L [11].

To construct an epitope-tagged versions of NAM9 and NAM9-1 first the 448 bp BamHIEcoRI fragment of pH13 was transferred to Bluescript vector (Stratagene) and then the sequence encoding epitope from influenza virus hemagglutinin protein (HA-epitope) was introduced using site-directed mutagenesis with the oligonucleotide 5'TGC AAC AAT ACA CGA CGA TCA AGC GTA GTC TGG GAC GTC GTA TGG GTA TTT
ACC ATT TCT CAA GTA3'. The AatII restriction site created with this procedure was used for selection and appropriate insertion of the HA epitope was finally confirmed by DNA sequencing. 3 ' Terminal BamHI-SalI fragment of NAM9 (or NAM9-1) sequence inserted in pH13, pAD6, pFL-NAM9 and pFL-NAM9-1 were substituted with the BamHI-SalI fragment containing the HA epitope created as described above, resulting in $\mathrm{pH} 13 / \mathrm{HA}, \mathrm{pAD} 6 / \mathrm{HA}$ centromeric and $\mathrm{pFL}$ NAM9/HA, pFL-NAM9-1/HA multicopy plasmids, respectively.

mRNA isolation and hybridisation. Cells of KL14-4A or its $r h o^{\circ}$ derivative were grown to the mid-log phase in liquid YPG or YPGALA medium. MB43-15C cells harbouring appropriate plasmid were grown to midlog phase in WO-ura and WO-leu medium. The harvested cells were broken with glass beads and RNA was isolated by phenol extraction. RNA, $10 \mu \mathrm{g}$, was loaded on $1.5 \%$ agarose gel in $1 \times$ Mops, $6.3 \%$ formaldehyde buffer, electrophoresed overnight and capillary transferred in $20 \times$ SSC to Hybond-C extra membrane (Amersham Manual Instructions). Hybridisation was carried out at $42^{\circ} \mathrm{C}$. The EcoRI-HindIII or AvaI-HindIII restriction fragments of the NAM9 gene and the XhoI-HindIII fragment of the yeast actin gene $A C T 1$, used as probes, were isolated from agarose by electroelution and labelled with $\left[\alpha^{32} \mathrm{P}\right] \mathrm{ATP}$ using the random prime system (Amersham).

Immunoblot analysis. Cells of W303-1B strain or its $r h 0^{\circ}$ derivative harbouring appropriate plasmid encoding HA-tagged Nam9p (or Nam9-1p) were grown to mid-log phase $\left(A_{600}=1\right)$ in liquid WO-ura glucose or galactose medium supplemented with appropriate amino acids. The total proteins ( $35 \mu \mathrm{g}$ ) extracted from trichloroacetic acid-treated cells were resolved by $10 \%$ polyacrylamide/SDS gel electrophoresis and electrophoretically transferred to Hybond-C extra membrane (Amersham). Membrane was blocked in 5\% skim milk powder in TBST buffer (10 mM Tris/HCl, pH $8.0,150 \mathrm{mM}$ $\mathrm{NaCl}, 0.05 \%$ Tween 20 ) for $30 \mathrm{~min}$ at room temperature. Mouse 12CA5 monoclonal antibody, specific for HA epitope (purchased from Berkeley Antibody Company) was 
added at a 1:2000 dilution, and incubation was carried out overnight at room temperature. Blot was washed in $5 \%$ skim milk powder in TBST buffer and incubated for $2 \mathrm{~h}$ with goat anti-mouse antibody conjugated to alkaline phosphatase (DAKO) at a 1:2000 dilution. Alkaline phosphatase was detected with nitro blue tetrazolium (NBT) and 5-bromo-4chloro-3-indolyl-1-phosphate (BCIP) (Promega).

Quantitation of RNA and protein. The levels of mRNA and protein on Northern and Western blots, respectively, were measured by scanning densitometry using Ultrascan XL Enhanced Laser Densitometer (LKB). NAM9 mRNA levels were normalised to actin mRNA levels.

\section{RESULTS AND DISCUSSION}

\section{Effect of gene dosage on NAM9 expression}

Extra functional copies of NAM9 were introduced into yeast cells using multicopy plasmids, derivatives of Yep351 [10] and pFL44L [11] (see Materials and Methods).

Total cellular RNA was analysed by Northern blotting using two independent NAM9 fragments as probes (see Materials and Methods). Both probes revealed the same transcript of $1.8 \mathrm{~kb}$. The size of the transcript is consistent with the size of the NAM9 ORF and the location of putative initiation and termination signals.

The amount of NAM9-1 mRNA in cells transformed with Yep351-NAM9-1 was approximately 32 times higher than in control cells transformed with Yep351 vector alone (Fig. 1A). This result is similar to those obtained for other mitoribosomal proteins: transcription was increased in proportion to the gene dosage $[12,13,14]$.

The relative amounts of Nam9p in cells containing different numbers of functional NAM9 copies is shown in Fig. 1B. For immunodetection of Nam9p we inserted a synthetic DNA sequence encoding an epitope from the influenza virus hemagglutinin protein (HA epitope) at the 3' end of the NAM9 gene, generating the pAD6/HA single copy and pFL-NAM9/HA multicopy plasmids (see
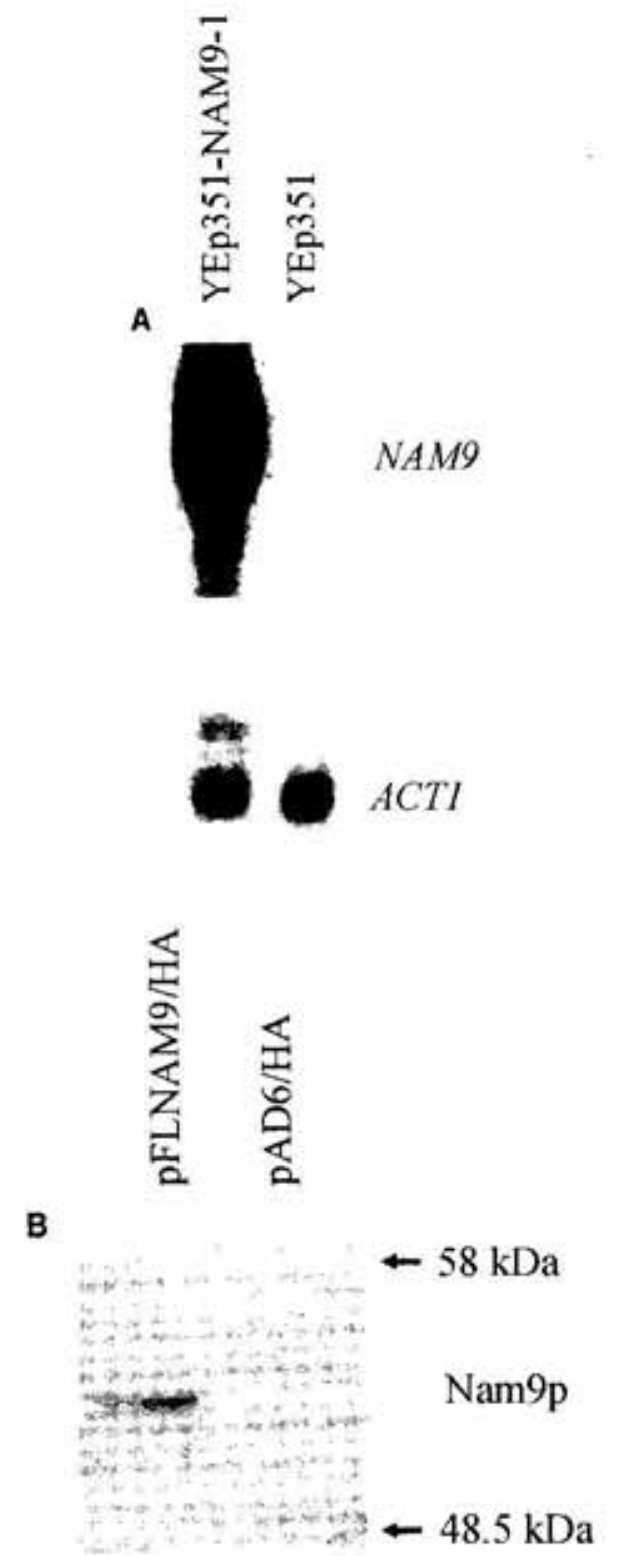

Figure 1. NAM9 expression in strains with elevated $N A M 9$ gene dosage.

A. Northern analysis. RNA samples were prepared from MB43-15C cells transformed with either YEp351NAM9-1 (Yep351-derivative carrying NAM9-1 gene) or Yep351. Cells were grown in minimal glucose WO-leu medium. Total RNA ( $10 \mu \mathrm{g}$ per lane) was separated by electrophoresis, transferred to a membrane and hybridised with the ${ }^{32} \mathrm{P}$-labelled AvaI-HindIII fragment from the NAM9 coding region. As a control for RNA loading, the same blot was hybridised with the ${ }^{32} \mathrm{P}-\mathrm{la}$ belled XhoI-HindIII fragment of the yeast ACT1 gene encoding actin. B. Western analysis. Protein samples were prepared from W303-1B cells transformed with either the multicopy pFL-NAM9/HA or the centromeric pAD6/HA plasmid carrying an HA-epitope tagged version of the NAM9 gene. Cells were grown in minimal WO-ura galactose medium. Samples of total cell protein (35 $\mu \mathrm{g}$ per lane) were subjected to immunoblot analysis with the HA-specific $12 \mathrm{CA} 5$ antibody, as described in Materials and Methods. 
Materials and Methods). The resulting fusion protein Nam9p-HA, expressed from the plasmid, restored growth phenotypes of mutants in the same fashion as did plasmid-expressed Nam9p (data not shown). This indicated that the presence of the HA epitope tag did not impede the function of Nam9p.

Extracts from cells bearing pAD6/HA and pFL-NAM9/HA plasmids were subjected to SDS/PAGE and subsequent immunoblot analysis. A band of about $53 \mathrm{kDa}$ corresponding to the Nam9p-HA fusion protein was detected with an antibody specific for HA. The level of Nam9p-HA expressed from the single copy plasmid, pAD6/HA, was below detection limit, whereas when overexpressed from pFL-NAM9/HA it was readily detected (Fig. 1B). No signal was observed in the control cells transformed with pAD6 plasmid or pFL44L vector (not shown). These data show that the steady-state level of Nam9p-HA was increased in the cells harbouring multiple copies of the NAM9-HA gene. This result strongly suggests that expression of Nam9p from the multicopy plasmid is not co-ordinated with the level of expression of other mitochondrial ribosomal proteins. Nam9p seems to be relatively stable in an unassembled form and accumulates in the cell if its rate of synthesis is increased. Another mitoribosomal protein, Mrp1p, also accumulates in the cell when overexpressed from a multicopy plasmid [15]. Expression of other mitoribosomal genes, however, seems to be co-ordinated probably by degradation of proteins synthesised in excess. In the case of $M R P 7$ overdose, accumulation of mRNA, but not protein, is observed [13].

\section{Effect of mitochondrial DNA on expres- sion of the NAM9 gene}

The expression of wild type NAM9 was analysed in isonuclear yeast strains with and without mitochondrial genotypes. The results of Northern and Western blot analyses are shown in Fig. 2. The level of the NAM9 transcript is two times higher in $r h o^{\circ}$ cells. The level of Nam9 protein is comparable in $r h o^{+}$and $r h o^{\circ}$ cells (we consider the small difference observed, below $30 \%$ as not signifi- cant). Expression of Nam9p in the $r h o^{\circ}$ cells indicates that it is not linked to expression of mitochondrially-encoded $\mathrm{mt}$ rRNAs.

Regulatory effects connected with the presence/absence of products encoded in the mtDNA on the expression of nuclear-encoded mitoribosomal proteins are diverse. Some genes (MRPL16, MRP7) are not differentially transcribed in $r h o^{+}$and $r h o^{\circ}$ cells [13, 16]. Transcription of MRP20, MRP49, MRP2, MRP13 genes $[12,17,18]$ is elevated in $r h o^{\circ}$ cells, which is consistent with the retrograde mode of regulation [19], but proteins are often absent in $r h o^{\circ}$ cells, even if

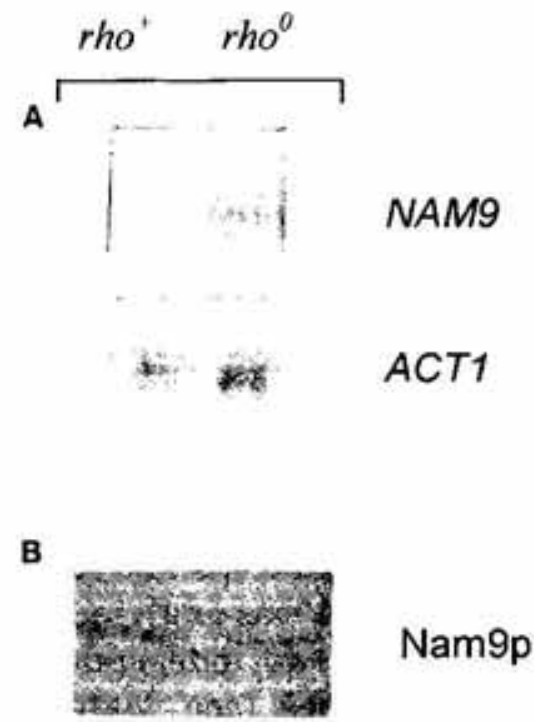

Figure 2. NAM9 expression in isonuclear strains with different mitochondrial genomes.

A. Northern analysis. RNA samples were prepared from strains KL14-4A $r h o^{+}$and KL14-4A $r h o^{\circ}$ growing in YPG medium. Hybridisation was carried out as described in legend to Fig. 1. B. Western analysis. Protein samples were prepared from W303-1B rho ${ }^{+}$ and W303-1B $r h o^{\circ}$ cells transformed with the pFL-NAM9/HA plasmid. Cells were grown in minimal WO-ura galactose medium. Immunoblot analysis was carried out as described in legend to Fig. 1.

their respective transcripts levels are elevated [18]. This is probably due to degradation of proteins when assembly of mitoribosomes is impossible in the absence of mitochondrial rRNAs. Other proteins, Mrp1p and Mrp7p, similarly to Nam9p accumulate in $r h o^{\circ}$ cells at a level comparable to that observed in the isogenic $r h o^{+}$cells. Therefore Nam9p belongs to the category of mitoribosomal proteins whose expression is not linked to other mitoribosomal components - 
whether to nuclear-encoded proteins or mitochondrial-encoded rRNAs.

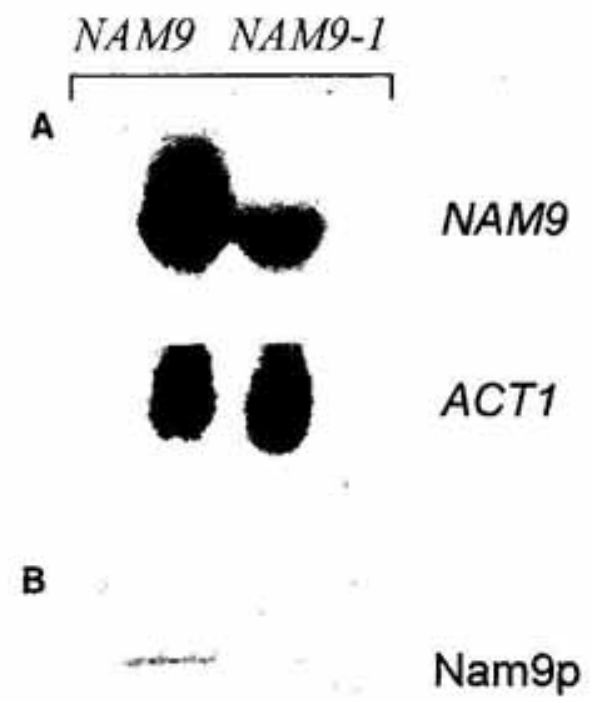

Figure 3. Expression of wild type and mutated Nam9p.

A. Northern analysis. Total RNA was isolated from the strain MB43-15C transformed with the centromeric plasmids pAD6 and pH13 carrying the wild type NAM9 and the mutated NAM9-1 allele, respectively. Cells were grown in minimal WO-ura glucose medium. RNA was hybridised to the NAM9 probe, as was described in legend to Fig. 1. As a control for RNA loading the same blot was hybridised with the actin probe (ACT1). B. Western analysis. W303-1B cells harbouring pFL-NAM9/HA or the pFL-NAM9-1/HA plasmid, carrying the epitope-tagged NAM9 alleles, were grown in WOura medium supplemented with $2 \%$ galactose. Immunoblot analysis was carried out as described in legend to Fig. 1.

\section{The level of Nam9p is affected by the} NAM9-1 missense mutation

The NAM9-1 mutation is a single amino acid substitution Ser82 $\rightarrow$ Leu in the region homologous to the ribosomal protein $\mathrm{S} 4$ from Escherichia coli. This mutation affects growth on media containing nonfermentable carbon sources and acts as a nuclear suppressor of some mitochondrial mit ochre mutants $[6,7]$. The amount of the transcript was determined for two transformants of NAM91 mutant: with single copy plasmid carrying the wild type and analogous plasmid carrying the mutated NAM9 gene (pAD6 and $\mathrm{pH}$ 13, respectively) (Fig. 3A). The level of the mRNA was 2.5 times higher for the transformant harbouring the wild type gene. The copy number of $\mathrm{pH} 13$ and $\mathrm{pAD} 6$ were the same as was confirmed by hybridisation to an URA3-specific probe (not shown). A slight decrease of the NAM9 transcript was also observed in the originally isolated NAM9-1 mutant, as compared with the wild type isogenic strain (Murawski, unpublished). The mechanism leading to the different mRNA levels is unknown. It is possible that the mutation specifically changes the structure of NAM9 mRNA and affects its stability. Several examples of mRNA-destabilising sequences within ORF have been described [20].

The wild type Nam9p accumulated in the cells is 9.7 times higher (Fig. 3B) as compared to mutated Nam9-1p. Our observations lead to the conclusion that overexpressed wild type protein is more abundant than the overexpressed mutated protein. We can only speculate about the bearing of these results on the physiological level of mutated Nam9$1 p$ in the cell.

\section{Sequence analysis of the $5^{\prime}$ upstream re- gion of the NAM9 gene: looking for possi- ble regulatory elements}

The principal function of the mitochondrial translation system in yeast is to synthesise several subunits of the respiratory enzyme complexes, therefore the synthesis of mitoribosomal proteins is expected to be repressed during non-respiratory growth in the presence of glucose. In fact glucose repression is a common feature in the regulation of nuclear genes coding for mitochondrial proteins. No common cis-acting sequence controlling the synthesis of mitoribosomal proteins has been found by computer search of the available $M R P$ genes [21]. The role of the HAP2/3/4 complex in the co-ordination of MRP transcription has been discussed [18]. About half of the $M R P$ genes, including $M R P 1,2,-L 6,7$, $13,-L 13,20,49$ and $Y M R 26$ which are known to respond to glucose repression, have 5' sequences that matched to the HAP2/3/4 consensus sequence, although this match could be not perfect $[5,18,21]$.

The sequence 5 '-TGATTGAT- 3 ' found at position -234 to -226 upstream of the AUG in the NAM 9 gene differs from the HAP2/3/4 consensus TNPuTTGGT by one nucleotide. 
Therefore the HAP2/3/4 complex could be involved in the regulation of NAM9 transcription. Surprisingly a sequence $-220\left(5^{\prime}\right.$ CGTTAAGCACGAT-3')-208, localised close to the putative HAP2/3/4 motif in the NAM9 gene, matches perfectly the consensus sequence of the $A B F 1$ transcription factor (CGTNNNNNPuPyGAPy). ABF1 has been described as a multifunctional DNA binding protein involved in coupling of the rate of mitochondrial biosynthesis to cellular growth [22] as well as in the co-ordination of cytosolic ribosomal protein synthesis [2]. Close localisation of HAP2/3/4 and ABF1 binding sites was detected previously in the promoter region of the $Q C R 8$ gene encoding a subunit of the mitochondrial ubiquinol-cytochrome oxidase [23, 24]. ABF1 is likely to interact with the HAP2/3/4 complex influencing the basal and induced NAM9 transcription levels. Identification of $\mathrm{ABF} 1$ and HAP2/3/4 consensus sequences as possible regulatory elements is consistent with the mitoribosomal character of the Nam9p.

\section{Glu Gal}

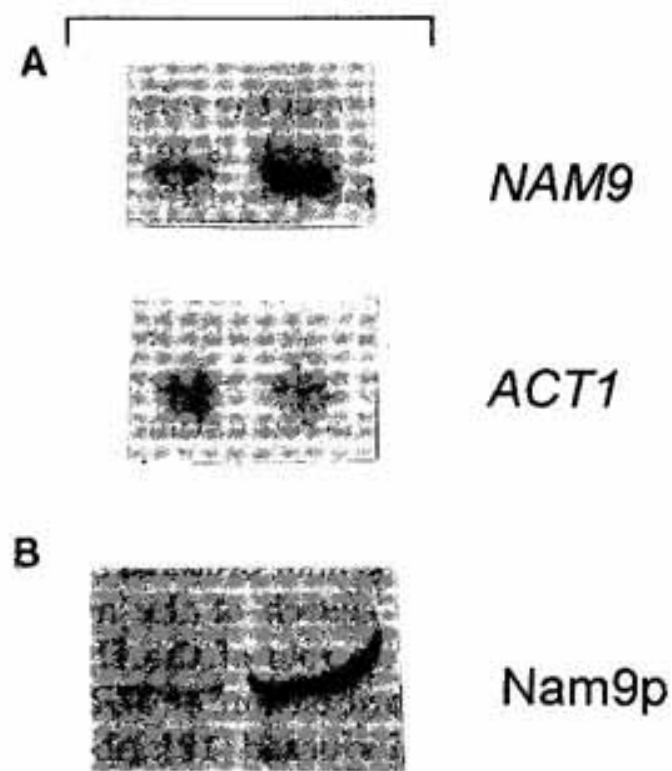

Figure 4. Catabolite repression of NAM9.

A. Northern analysis. Yeast strain KL14-4A was grown in YPG $2 \%$ glucose (Glu) or YPGALA $2 \%$ galactose (Gal) medium. Hybridisation was carried out as described in legend to Fig. 1. B. Western analysis. Yeast strain W303-1B harbouring the pFL-NAM9/HA plasmid, carrying the epitope-tagged NAM9 gene, was grown in WO-ura medium supplemented with appropriate $2 \%$ glucose (Glu) or $2 \%$ galactose (Gal). Immunoblot analysis was carried out as described in legend to Fig. 1.
NAM9 expression is controlled by carbon source

Steady state levels of the NAM9 mRNA and protein were determined in the wild type cells growing under catabolite repression in $2 \%$ glucose and in derepressed conditions in $2 \%$ galactose. The results of Northern and Western blot analyses are shown in Fig. 4 . The level of NAM9 transcript was 2.9 times higher in derepressed cells (Fig. 4A), thus the transcription of this gene appears to be regulated by glucose repression. The response to carbon source was even more pronounced for the Nam9 protein levels (Fig. 4B). The difference was sevenfold implicating a postranscriptional component in the regulation of the NAM9 expression.

All twelve mitochondrial ribosomal proteins studied so far in respect of the control of gene expression were regulated to various extent in response to glucose (for references see [5]). Although the primary control seems to occur at the level of transcription, the differences in protein levels often are higher than those observed for the corresponding transcripts. This indicates a posttranscriptional modulation of the response to catabolite repression. The most pronounced is the case of the $M R P 7$ gene, regulated by carbon source only at the level of translation [13].

We thank Dr J. Smagowicz for scanning the blots. We are grateful to Professor P.P. Słonimski and Dr A. Dmochowska for helpful discussions and comments.

\section{REFERENCES}

1. Nomura, M.R., Gourse, R. \& Baughman, G. (1984) Regulation of the synthesis of ribosomes and ribosomal components. Annu. Rev. Biochem. 53, 75-117.

2. Goncalves, P.M., Griffioen, G., Minnee, R., Bosme, M., Kraakman, L.S., Mager, W. \& Planta, R.J. (1995) Transcription activation of yeast ribosomal protein genes requires additional elements apart from binding sites of Abflp or Rap1p. Nucleic Acids Res. 23, 1475-1480. 
3. Woolford, J.L. \& Warner, J.R. (1991) The ribosome and its synthesis; in The Molecular Biology of the Yeast Saccharomyces (Broach, J.R., Pringle, J.R. \& Jones, E.W., eds.) Cold Spring Harbor University Press.

4. Kitakawa, M. \& Isono, K. (1991) The mitochondrial ribosomes. Biochimie 73, 813-825.

5. Grohmann, L., Kitakawa, M., Isono, K., Goldschmidt-Resin, S. \& Graack, H.R. (1994) The yeast nuclear gene MRP.L13 codes for a protein of the large subunit of the mitochondrial ribosome. Curr. Genet. 26, 8-14.

6. Boguta, M., Dmochowska, A., Borsuk, P., Wróbel, K., Gargouri, A., Lazowska, J., Slonimski, P.P., Szczesniak, B. \& Kruszewska, A. (1992) NAM9 nuclear suppressor of mitochondrial ochre mutations in Saccharomyces cerevisiae codes for a protein homologous to $\mathrm{S} 4$ ribosomal protein from chloroplasts, bacteria and eukaryotes. Mol. Cell. Biol. 12, 402-412.

7. Dmochowska, A., Konopińska, A., Krzymowska, M., Szcześniak, B. \& Boguta, M. (1995) The NAM9-1 suppressor mutation in a nuclear gene encoding ribosomal mitochondrial protein of Saccharomyces cerevisiae. Gene 162, 81-85.

8. Dujardin, G., Pajot, P., Groudinsky, O. \& Slonimski, P.P. (1980) Long range control circuits within mitochondria and between nucleus and mitochondria. Mol. Gen. Genet. 179, 469-482.

9. Konopińska, A., Szcześniak, B. \& Boguta, M. (1995) Nucleotide sequence of the GDS1 gene of Saccharomyces cerevisiae. Yeast 11, 1513-1518.

10. Hill, J.E., Myers, A.M., Koerner, T.J. \& Tzagoloff, A. (1986) Yeast/E. coli shuttle vectors with multiple unique restriction sites. Yeast 2, 163-167.

11. Boneaud, N., Ozier-Kalogeropoulos, O., Li, G., Labousse, M., Minnele-Sebastia, L. \& Lacroute, F. (1991) A family of low and high copy replicative, integrative and single-stranded $S$. cerevisiae / E. coli shuttle vectors. Yeast 7, 609-615.

12. Partaledis, J.A. \& Mason, T.L. (1988) Structure and regulation of a nuclear gene in Sac- charomyces cerevisiae that specifies MRP13, a protein of the small subunit of the mitochondrial ribosome. Mol. Cell. Biol. 8, 3647-3660.

13. Fearon, K. \& Mason, T. (1988) Structure and regulation of a nuclear gene in Saccharomyces cerevisiae that specifies MRP7, a protein of the large subunit of the mitochondrial ribosome. Mol. Cell. Biol. 8, 3636-3646.

14. Kang, W., Matsuhita, Y. \& Isono, K. (1991) Cloning and analysis of YMR26, the nuclear gene for mitochondrial ribosomal protein in Saccharomyces cerevisiae. Mol. Gen. Genet. 225, 474-482.

15. Dang, H., Franklin, G., Darlak, K., Spatola, A.F. \& Ellis, S.R. (1990) Discoordinate expression of the yeast mitochondrial ribosomal protein MRP1. J. Biol. Chem. 265, 7449-7454.

16. Pan, J. \& Mason, T. (1995) Identification of the yeast nuclear gene for the mitochondrial homologue of bacterial ribosomal protein L16. Nucleic Acids Res. 18, 3673-3677.

17. Myers, A.M., Crivellone, M.D. \& Tzagoloff, A. (1987) Assembly of the mitochondrial membrane system: $M R P 1$ and $M R P 2$, two yeast nuclear genes coding for mitochondrial ribosomal proteins. J. Biol. Chem. 262, 3388-3397.

18. Fearon, K. \& Mason, T. (1992) Structure and function of MRP20 and MRP49, the nuclear genes for two proteins of the $54 \mathrm{~S}$ subunit of the yeast mitochondrial ribosome. J. Biol. Chem. 267, 5162-5170.

19. Liao, X. \& Butow, R. (1993) Two yeast genes required for a novel path of communication

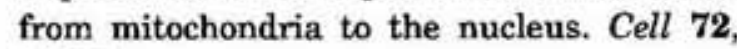
61-71.

20. Sachs, A.B. (1993) Messenger degradation in eukaryotes. Cell 74, 413-421.

21. Harrer, R., Schwank, H.-J. \& Schweizer, E. (1993) Molecular cloning and analysis of the nuclear gene MRP-L6 coding for a putative mitochondrial ribosomal protein from Saccharomyces cerevisiae. Curr. Genet. 24, 136-140.

22. Grivell, L.A., Dorsman, J.C., Gozdzicka-Jozefiak, A., Van Heeswijk, W.C. \& de Winde, J.H. (1990) Generalised growth control of mito- 
chondrial biogenesis in yeast; in Structure, Function and Biogenesis of Energy Transfer Systems (Quaghiarello, E., Papa, S., Palmieri, F., Saccone, C., eds.) Elsevier, Den Haag, The Nederlands.

23. de Winde, J.H. \& Grivell, L.A. (1992) Global regulation of mitochondrial biogenesis in $\mathrm{Sac}$ charomyces cerevisiae: $\mathrm{ABF} 1$ and $\mathrm{CPF} 1$ play opposite roles in regulating expression of the
QCR8 gene, which encodes subunit VIII of the mitochondrial ubiquitinol-cytochrome $c$ oxidoreductase. Mol. Cell. Biol. 12, 2872-2883.

24. de Winde, J.H. (1992) Global Regulation of Mitochondrial Biogenesis in the Yeast Saccharomyces cerevisiae. $\mathrm{Ph} . \mathrm{D}$. Thesis, University of Amsterdam. 